\title{
Supplemental Information: Wigner time delay induced by a single quantum dot
}

\author{
Max Strauß, ${ }^{1}$ Alexander Carmele, ${ }^{2}$ Julian Schleibner, ${ }^{2}$ Marcel Hohn, ${ }^{1}$ Christian \\ Schneider, ${ }^{3}$ Sven Höfling, ${ }^{3,4}$ Janik Wolters, ${ }^{1,5}$ and Stephan Reitzenstein ${ }^{1}$ \\ ${ }^{1}$ Insitut für Festkörperphysik, Technische Universität Berlin, D-10263 Berlin, Germany \\ ${ }^{2}$ Institut für Theoretische Physik, Technische Universität Berlin, D-10263 Berlin, Germany \\ ${ }^{3}$ Technische Physik, Physikalisches Institut,Wilhelm Conrad Röntgen Center for Complex Material Systems, \\ Universität Würzburg, D-97074 Würzburg, Germany \\ ${ }^{4}$ SUPA, School of Physics and Astronomy, University of St. Andrews, St. Andrews KY16 9SS, United Kingdom \\ ${ }^{5}$ Present address: Department of Physics, University of Basel, \\ Klingelbergstrasse 82, CH-4056 Basel, Switzerland
}

(Dated: February 20, 2019)

\section{S-I. SAMPLE TECHNOLOGY}

The experiment is based on self-assembled semiconductor InGaAs quantum dots (QDs) grown by molecular beam epitaxy (MBE). The QDs with an Indium content of $60 \%$ are embedded in a GaAs host matrix and were grown by the Stranski-Krastanov growth mode at a temperature of $520{ }^{\circ} \mathrm{C}$. Here, the Indium flush technique [1] was applied to blue-shift the emission by precisely controlling the height of the QDs. The QDs have a density of about $10^{9} \mathrm{~cm}^{-2}$ and the height and lateral dimensions of the modulation doped QDs are about $2 \mathrm{~nm}$ and $20 \mathrm{~nm}$, respectively. The ensemble emission is centered at approximately $927 \mathrm{~nm}$ and the typical biexciton-exciton binding energy is in the range of 1-3 $\mathrm{meV}$. To enhance the photon extraction efficiency the QD layer is embedded the center of the one- $\lambda$ thick GaAs cavity of a planar low-Q distributed Bragg reflector (DBR) cavity consisting of 24 lower and 5 upper mirror pairs. In addition to the improved directionality obtained by embedding the QDs into the planar microcavity, additional increase of their photon extraction efficiency up to $42 \%$ is achieved for QDs which self-align to Gaussian shaped nanohill defects that naturally form during the epitaxial growth [2]. A typical micro-photoluminescence $(\mu \mathrm{PL})$ spectrum of the sample recorded at $5 \mathrm{~K}$ and an excitation power of $140 \mathrm{nW}$ is presented in Fig. S1. It shows a strong single-QD line at approximately $924.9 \mathrm{~nm}$ which was used for investigating the Wigner time delay under pulsed resonant excitation in the main text.

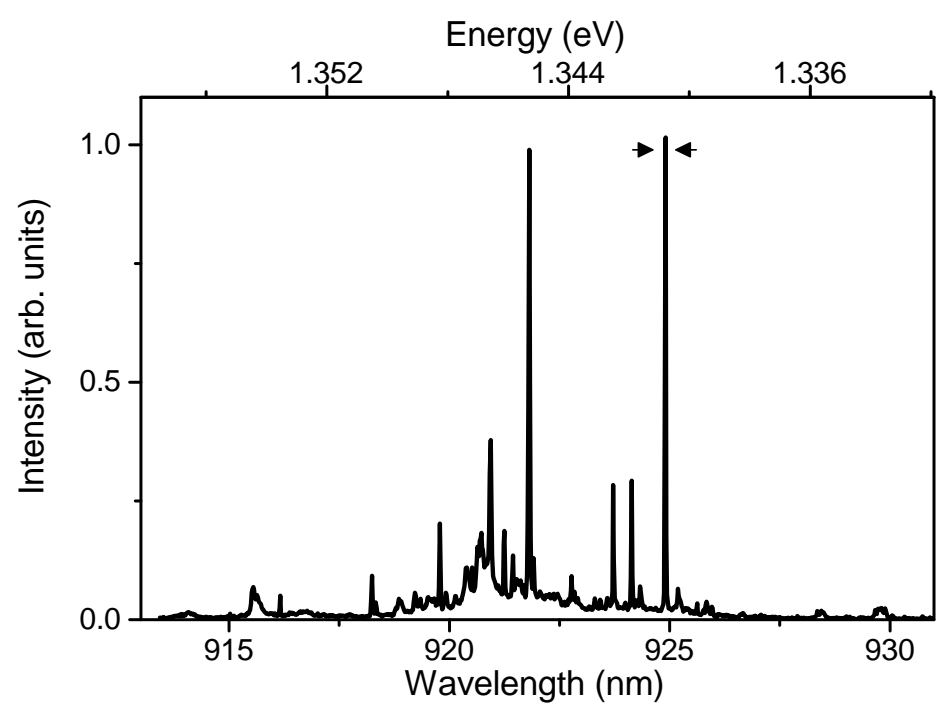

FIG. S1. Low-excitation $\mu \mathrm{PL}$ spectrum of the sample under study. The spectrum shows single QD emission lines with the ensemble emission centered at about $923 \mathrm{~nm}$. The sharp and strong emission line at approximately $924.9 \mathrm{~nm}$ (indicated by arrows) is the emission line of the QD used for investigating the Wigner time delay in the main manuscript. 


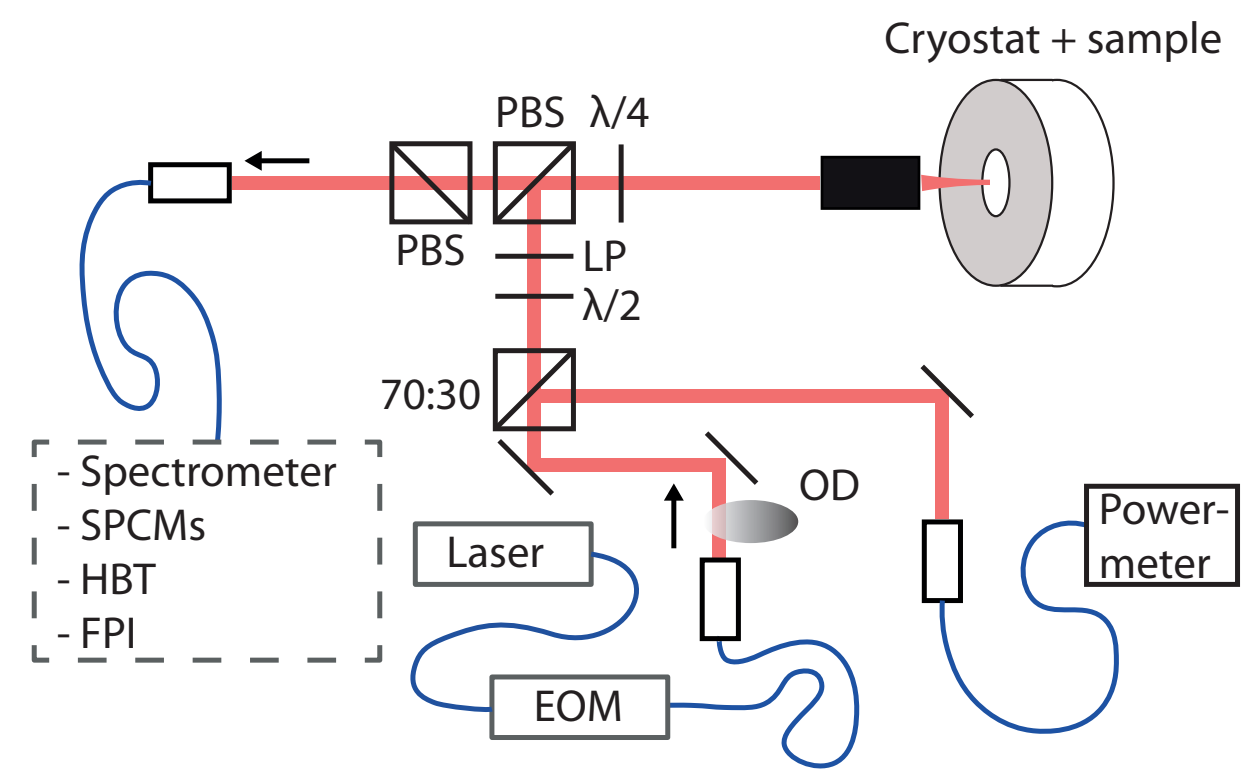

FIG. S2. Sketch of the experimental setup. Abbreviations: EOM - electrooptical modulator, FPI - Fabry-Perot interferometer, HBT - Hanbury Brown and Twiss interferometer, LP - linear polariser, $\lambda / 2$ - half wave plate, $\lambda / 4$ - quarter wave plate, OD - optical density filter, PBS - polarising beam splitter, SPCM - single-photon counting module.

\section{S-II. EXPERIMENTAL SETUP}

The QD is excited by a tunable resonant $\mathrm{CW}$ diode laser and a weak non-resonant diode laser $(\lambda=785 \mathrm{~nm})(\mathrm{not}$ shown in Fig. S2). The fiber coupled resonant and non-resonant laser are superimposed using a fiber beam splitter. The amplitude of the resonant laser can be temporally modulated using a fiber based electro-optical modulator (EOM) with an extinction above 1:1000. The EOM is controlled by a customized programmable pulse generator allowing for arbitrary pulse shapes with a maximum achievable length of $\Delta t=1.05 \mathrm{~ns}$ (FWHM). The light is then coupled to free space as a collimated beam. To ensure a high degree of linear polarization the laser beam passes a half-wave plate $(\lambda / 2)$ and a linear polarizer (LP) prior to being reflected by a polarizing beam splitter (PBS) towards the sample. The beam is focused onto the sample using a microscope objective $(\mathrm{NA}=0.65)$. The sample itself is mounted inside a helium-flow cryostat and cooled to a temperature of $5 \mathrm{~K}$. The fluorescence is collected by the same microscope objective. The reflected laser and fluorescence are separated by polarization filtering using two PBSs that transmit light orthogonal to the excitation and by focusing the collected light onto the facet of a single-mode fiber prior to photodetection. The luminescence is detected by a single-photon counting module and photon counts are histogrammed using a time-correlated single-photon counting (TCSPC) electronics with an overall timing resolution of $\Delta \tau_{\mathrm{FWHM}}=390 \mathrm{ps}$.

\section{S-III. RESONANCE FLUORESCENCE EMISSION SPECTRA OF A QUANTUM DOT TWO-LEVEL SYSTEM}

In this section we present resonance fluorescence emission spectra of the QD under study in the main text. In a system without pure dephasing processes, the emission of the TLS is dominated by coherent scattering processes in the low excitation regime while the resonance fluorescence signal is exclusively incoherent in the high excitation regime. The resonance fluorescence emission spectrum of a TLS in these two regimes is also markedly different as is illustrated by the emission spectra in Fig. S3. For high excitation powers (upper panel) we observe the Mollow triplet $\left(P=130 P_{\text {sat }}\right)$ which collapses into one single peak for a power below saturation $\left(P=0.14 P_{\text {sat }}\right)$ (lower panel). In principle, the emission spectrum under low excitation should only reveal the instrument response function of our scanning Fabry-Perot interferometer (FPI) which is $436 \mathrm{neV}(105 \mathrm{MHz})$. We observe, however, an emission linewidth of $0.84 \mu \mathrm{eV}$ which is below the theoretical limit of the incoherent spectrum of $2 / T_{2}=2.96 \mu \mathrm{eV}$ but above the resolution limit of our FPI. Therefore, this measurement also evidences that we find a substantial amount of incoherently scattered photons in our signal even at low excitation powers due to the presence of pure dephasing. The emission spectrum at low excitation powers is thus a weighted superposition of the coherent and incoherent 


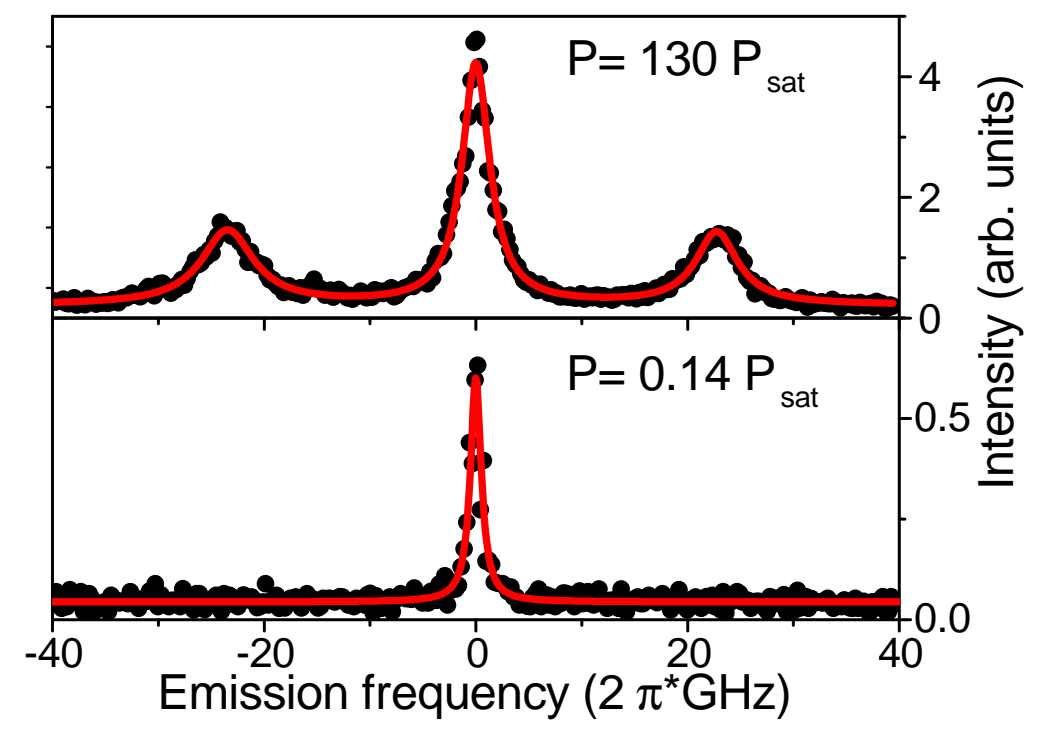

FIG. S3. Resonance fluorescence emission spectra below (bottom) and above saturation (top). At high excitation powers the emergence of sidebands is evidence of the resonant interaction between coherent light field and TLS. The red lines are Lorentzian fits to the data.

contribution.

\section{S-IV. THEORETICAL MODELLING VIA NON-MARKOVIAN LINDBLAD FORMALISM}

It is known that lattice vibrations in semiconductor nanostructures give rise to new effects not known in typical atomic quantum optics and which are not included in Markovian treatments of the semiconductor environment such as phonon-mediated off-resonant cavity feeding [5, 6], formation of phonon-assisted Mollow triplets [7, 8], phononassisted Rabi oscillations [9, 10] and exciting reservoir correlation length in Hong-Ou-Mandel type of experiments [11, 12]. These features stem from the non-Ohmian spectral density of the semiconductor lattice vibrations. Lindbladrelated master equation treatments usually do not include these features and therefore overestimate the impact of dephasing. Here, we model the dynamics via a Born-factorization, and cluster-expansion approach which is valid up to a temperature of $50 \mathrm{~K}$ and in the weak-driving limit. Beyond this limit, an extended Lindblad-approach can be employed [13] but our system under study at cryogenic temperatures of about $5 \mathrm{~K}$ is well-described in the factorization approach. The Hamiltonian of the system in the Born-Oppenheimer limit reads:

$$
H / \hbar=\left(\omega_{g}-\omega_{L}\right) a_{c}^{\dagger} a_{c}+\Omega(t)\left(a_{v}^{\dagger} a_{c}+a_{c}^{\dagger} a_{v}\right)+\sum_{\mathbf{q}} \omega_{q} b_{\mathbf{q}}^{\dagger} b_{\mathbf{q}}+\sum_{q}\left(g_{v c}^{\mathbf{q}} b_{\mathbf{q}}+g_{v c}^{\mathbf{q} *} b_{\mathbf{q}}^{\dagger}\right) a_{c}^{\dagger} a_{c}
$$

for a rotating frame in the laser frequency and detuning $\Delta=\omega_{g}-\omega_{L}$. The coupling to acoustic phonons is incorporated, and the linear dispersion reads: $\omega_{q}=c_{s}|\mathbf{q}|$. The coupling element is the deformation potential. The laser excitation reads

$$
\Omega(t)=\Omega_{L} \sqrt{\frac{\pi}{\left(2 \tau^{2}\right)}} \exp \left[-\frac{\left(t-t_{0}\right)^{2}}{2 \tau^{2}}\right]
$$

with the amplitude $\Omega_{L}$. The semiconductor Bloch equation are solved in the Heisenberg picture. The one-electron assumption is assumed $\left\langle a_{v}^{\dagger} a_{v}\right\rangle=1-\left\langle a_{c}^{\dagger} a_{c}\right\rangle$. The phonon dynamics is treated non-Markovianly in the bath assumption limit, i.e. second-order Born (approximation?). The set of equations of motion reads (including a Markovian radiative 
decay constant $\Gamma)$ :

$$
\begin{aligned}
\partial_{t}\left\langle a_{c}^{\dagger} a_{c}\right\rangle & =-2 \Gamma\left\langle a_{c}^{\dagger} a_{c}\right\rangle+2 \operatorname{Im}\left[\Omega(t)\left\langle a_{v}^{\dagger} a_{c}\right\rangle\right] \\
\partial_{t}\left\langle a_{v}^{\dagger} a_{c}\right\rangle & =-(\Gamma+i \Delta)\left\langle a_{v}^{\dagger} a_{c}\right\rangle-i \Omega(t)\left(2\left\langle a_{c}^{\dagger} a_{c}\right\rangle-1\right)-i \sum_{\mathbf{q}} g_{v c}^{\mathbf{q}}\left\langle b_{\mathbf{q}} a_{v}^{\dagger} a_{c}\right\rangle+g_{v c}^{\mathbf{q} *}\left\langle b_{\mathbf{q}}^{\dagger} a_{v}^{\dagger} a_{c}\right\rangle \\
\partial_{t}\left\langle b_{\mathbf{q}} a_{v}^{\dagger} a_{c}\right\rangle & =-\left(\Gamma+i \Delta+i \omega_{q}\right)\left\langle b_{\mathbf{q}} a_{v}^{\dagger} a_{c}\right\rangle-i \Omega(t)\left(2\left\langle b_{\mathbf{q}} a_{c}^{\dagger} a_{c}\right\rangle-\left\langle b_{\mathbf{q}}\right\rangle\right)-i g_{v c}^{\mathbf{q} *}\left\langle b_{\mathbf{q}}^{\dagger} b_{\mathbf{q}}\right\rangle\left\langle a_{v}^{\dagger} a_{c}\right\rangle \\
\partial_{t}\left\langle b_{\mathbf{q}}^{\dagger} a_{v}^{\dagger} a_{c}\right\rangle & =-\left(\Gamma+i \Delta-i \omega_{q}\right)\left\langle b_{\mathbf{q}}^{\dagger} a_{v}^{\dagger} a_{c}\right\rangle-i \Omega(t)\left(2\left\langle b_{\mathbf{q}} a_{c}^{\dagger} a_{c}\right\rangle^{*}-\left\langle b_{\mathbf{q}}\right\rangle^{*}\right)-i g_{v c}^{\mathbf{q}}\left\langle b_{\mathbf{q}} b_{\mathbf{q}}^{\dagger}\right\rangle\left\langle a_{v}^{\dagger} a_{c}\right\rangle \\
\partial_{t}\left\langle b_{\mathbf{q}} a_{c}^{\dagger} a_{c}\right\rangle & =-\left(2 \Gamma+i \omega_{q}\right)\left\langle b_{\mathbf{q}} a_{c}^{\dagger} a_{c}\right\rangle-i \Omega(t)\left(\left\langle b_{\mathbf{q}} a_{v}^{\dagger} a_{c}\right\rangle-\left\langle b_{\mathbf{q}}^{\dagger} a_{v}^{\dagger} a_{c}\right\rangle^{*}\right)+i g_{v c}^{\mathbf{q} *}\left\langle b_{\mathbf{q}}^{\dagger} b_{\mathbf{q}}\right\rangle\left\langle a_{c}^{\dagger} a_{c}\right\rangle \\
\partial_{t}\left\langle b_{\mathbf{q}}\right\rangle & =-i \omega_{q}\left\langle b_{\mathbf{q}}\right\rangle-i g_{v c}^{\mathbf{q} *}\left\langle a_{c}^{\dagger} a_{c}\right\rangle .
\end{aligned}
$$

In the low temperature limit, the second-order factorization is still valid, and allows via the bath assumption to include stimulated phonon emission and absorption. The phonon occupation number are given via the Bose-Einstein distribution and depends on the temperature via:

$$
\left\langle b_{\mathbf{q}}^{\dagger} b_{\mathbf{q}}\right\rangle=\left[\exp \left(\hbar \omega_{q} /\left(k_{B} T\right)\right)-1\right]^{-1}
$$

The coupling element of the QD to the phonon continuum is determined via the deformation potential. This potential depends on the confinement energies of electrons and holes of the QDs, and on the semiconductor background material. We use the standard parameter of deformation coupling values of an approximate spherical QD. Phonon coupling element $\left|g_{v c}^{\mathbf{q}}\right|=\left|g_{v v}^{\mathbf{q}}-g_{c c}^{\mathbf{q}}\right|$, where

$$
g_{i}^{\mathbf{q}}=\sqrt{\frac{\hbar q}{2 \rho c_{s} V}} D_{i} e^{-\frac{q^{2} \hbar}{4 m_{i} \omega_{i}}} .
$$

Used parameters: sound velocity of GaAs $c_{\mathrm{s}}=0.00511 \mathrm{~nm} / \mathrm{fs}$, deformation potentials $D_{v}=-5.38 \mathrm{eV}, D_{c}=-11.68 \mathrm{eV}$, effective masses: $m_{c_{3}}=0.063, m_{v}=0.45$, confinement energies $\hbar \omega_{c}=0.040 \mathrm{eV}, \hbar \omega_{v}=0.02 \mathrm{eV}$ and mass density of GaAs $\rho=5370 \mathrm{~kg} / \mathrm{m}^{3}[6,14,15]$.

In the calculations, we fixed the radiative decay time to the experiment $\Gamma=600 \mathrm{ps}^{-1}$, and choose a time-scale, so we reproduce the maximum of the Wigner delay on resonance at $\Delta=0$, for a pulse width of $\tau=(10 \Gamma)^{-1}$ and a fixed pure dephasing for a given pulse area fixed at $\Omega_{L}=0.4$ for the detuning dependent parameter plot. This pulse area is changed but everything else is kept fixed for the power parameter plot on resonance.

\section{S-V. ON THE REPRODUCIBILITY OF THE WIGNER TIME DELAY OF A RESONANTLY DRIVEN QUANTUM DOT}

To demonstrate the robustness and reproducibility of the Wigner time delay reported in the main text. We studied a second QD of the same sample using the same experimental setup and similar experimental parameters such as the excitation power. Fig. S3 summarizes the results obtained for this QD. The experiments were performed at an excitation power of $3 \mathrm{nW}$ well below the saturation power of $82 \mathrm{nW}$. As can be seen in panel (a) of Fig. S4 the resonantly scattered light experience a similar delay with respect the excitation laser as for the QD discussed in the main text of the paper. A quantitative analysis reveals a detuning dependent Wigner time delay with a maximum delay of about 500 ps at zero detuning between the TLS and the excitation laser. Again, the theoretical description based on non-Markovian dynamics is in good agreement with the experimental data. Panel (c) presents the integrated intensity of the scattered pulses as a function of laser detuning to complement the data set. 

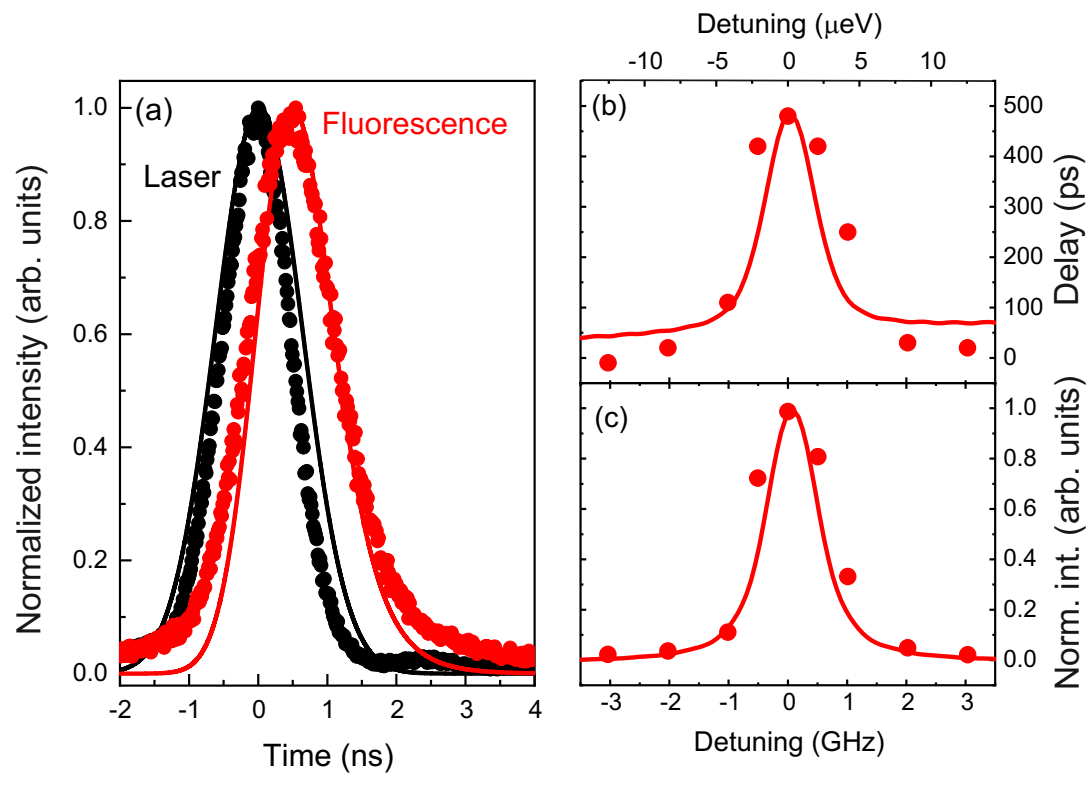

FIG. S4. Wigner time delay of a second QD. The QD was studied under the same experimental parameters than the one in the main text. It a Wigner time delay of about 500 ps under resonant excitation (see panel (a)) and the characteristic decrease of the time delay for increasing detuning between the TLS and the excitation laser (see panel (b). Panel (c) shows the intensity of the resonantly scattered light as a function of laser detuning. The experimental data is well described by theoretical curves (red lines) taking our non-Markovian model into account.

[1] J. M. Garcia, T. Mankad, P. O. Holtz, P. J. Wellman, and P. M. Petroff. Electronic states tuning of InAs self-assembled quantum dots. Appl. Phys. Lett., 72:3172, 1998.

[2] S. Maier, P. Gold, A. Forchel, N. Gregersen, J. Mørk, S. Höfling, C. Schneider, and M. Kamp. Bright single photon source based on self-aligned quantum dot-cavity systems. Opt. Express, 22:8136, 2014.

[3] C. Cohen-Tannoudji, J. Dupont-Roc, and G. Grynberg. Atom-Photon Interactions: Basic Processes and Applications. Wiley-VCH, 2004

[4] H. S. Nguyen, G. Sallen, C. Voisin, P. Roussignol, C. Diederichs, and G. Cassabois. Ultra-coherent single photon source. Appl. Phys. Lett., 99:261904, 2011.

[5] S. Hughes, P. Yao, F. Milde, A. Knorr, D. Dalacu, K. Mnaymneh, V. Sazonova, P. J. Poole, G. C. Aers, J. Lapointe, R. Cheriton, and R. L. Williams. Influence of electron-acoustic phonon scattering on off-resonant cavity feeding within a strongly coupled quantum-dot cavity system. Phys. Rev. B, 83:165313, 2011.

[6] U. Hohenester, A. Laucht, M. Kaniber, N. Hauke, A. Neumann, A. Mohtashami, M. Seliger, M. Bichler, and J. J. Finley. Phonon-assisted transitions from quantum dot excitons to cavity photons. Phys. Rev. B, 80:201311, 2009.

[7] S. M. Ulrich, S. Ates, S. Reitzenstein, A. Löffler, A. Forchel, and P. Michler. Dephasing of triplet-sideband optical emission of a resonantly driven InAs/GaAs quantum dot inside a microcavity. Phys. Rev. Lett., 106:247402, 2011.

[8] J. Kabuss, A. Carmele, M. Richter, and A. Knorr. Microscopic equation-of-motion approach to the multiphonon assisted quantum emission of a semiconductor quantum dot. Phys. Rev. B, 84:125324, 2011.

[9] A. Vagov, M. D. Croitoru, M. Glässl, V. M. Axt, and T. Kuhn. Real-time path integrals for quantum dots: Quantum dissipative dynamics with superohmic environment coupling. Phys. Rev. B, 83:094303, 2011.

[10] J. Förstner, C. Weber, J. Danckwerts, and A. Knorr. Phonon-assisted damping of Rabi oscillations in semiconductor quantum dots. Phys. Rev. Lett., 91:127401, 2003.

[11] A. Thoma et al. Exploring dephasing of a solid-state quantum emitter via time-and temperature-dependent Hong-OuMandel experiments. Phys. Rev. Lett., 116:033601, 2016.

[12] Hui Wang et al. Near-Transform-Limited Single Photons from an Efficient Solid-State Quantum Emitter. Phys. Rev. Lett., 116:213601, 2016.

[13] C. Roy and S. Hughes Polaron master equation theory of the quantum-dot Mollow triplet in a semiconductor cavity-QED system. Phys. Rev. B, 85:115309, 2016.

[14] A. Carmele, A. Knorr, and F. Milde. Stabilization of photon collapse and revival dynamics by a non-Markovian phonon bath. New J. Phys 15:105024, 2013. 
[15] A. Vagov, M. D. Croitoru, M. Glässl, V. M. Axt, and T. Kuhn Real-time path integrals for quantum dots: Quantum dissipative dynamics with superohmic environment coupling. Phys. Rev. B 83:094303, 2011. 